\title{
ECG (Electro Cardio Gram) Sensor IoT for on line T-Wavelet Analysis to Detect Medical Signal Deviation
}

\author{
Er M Seshagiri Rao, ${ }^{1}$ Dr V S Chouhan ${ }^{2}$, \\ Dr M Sai Lakshmi Lalita Devi BS, (MD) ${ }^{3}$ \\ ${ }^{1}$ B.Tech, MS, PG Dip in VLSI Engg, Sr Memb IEEE, Ministry of Electronics and IT, India \\ ${ }^{2}$ Faculty, MBM College, Dept of ECE JNV University, Rajasthan, India \\ ${ }^{3}$ Davao Medical College, Philippines
}

\begin{abstract}
G mobile technologies or LTE (Long Term Evolution) release 10 onwards, provision is kept for Internet of Things (IoT) data in the mobile communication technologies. Industrial IoT 4.0 under evolution is expecting every kind of sensor to be integrated thorough IoT, for it's connectivity with internet. Early detection of deviation in Electro Cardio Gram (ECG) has specific diagnostic and treatment advantage, in the case of Cardio Vascular Disease (CVD) patients, acquiring ECG frames of the beats continuously via. Mobile carried throughout. This type of warning and awareness to patients are critical in preventing permanent damage and saving the most part of the heart muscle. In order to apply Digital Signal Processing (DSP) algorithms on the ECG heart beats, the digitized ECG, from the body area electronics, based on ECG IoT Sensor design approach need to communicate to a mobile for wavelet analysis or in-turn to a doctor's central system via., it's Internet Protocol(IP). An algorithms to detect Deep T-Wave inversion is evolved, to provide early warning and plot the results on the mobile screen and to send this information to physician or cardiologist as and when required. The methodology adopted to acquire ECG from body area electronics sticked to body surface up to displaying it on the mobile screen, via. ECG IoT architecture has been illustrated.
\end{abstract}

Keywords: IoT, ECG, T-Wavelet, Android Graphview, Body Area Electronics

\section{Introduction}

The research trend has been to make every sensor, be it electrical, magnetic, chemical, optical, mechanical, sound, thermal etc, the evolving Internet of Things (IoT) based sensor. In this trend, Electro Cardiogram Gram (ECG) Sensor front end is not to be an exception, to acquire, heart muscle conduction based electrical signal. As part of the work under taken, ECG sensor evolved, based on IoT stack has systemic design advantage in acquiring digitized ECG potentials from the body, which represent integrated electrical action potentials originate from various heart muscles or anatomy. Hybrid electronics design is resorted, in order to have flexible epidermal patch at the front end [1]. Rest of the electronics is at considerable speeds required for Blue tooth or USB communication of the cellular mobile phone and internet protocol (IP) based packet communication by ECG IoT.

\section{IoT For ECG Diagnostic Monitoring}

Internet of Things (IoT) term was coined by Kevin Ashton of the Massachusetts Institute of Technology (MIT), at the time, when industrial automation technologies were starting to move from the factory into new environments like hospitals, banks and offices. As early Machine to Machine implementations grew increasingly, more sophisticated, machines were connected to other kinds of devices via. internet to computing systems and servers. While the Internet is a critical standardised, enabling element, it is only part of the essential concept - the idea to connect our real practical world, to virtual world of information systems, for transformation to smart connected systems and operations. IoT is an area, where embedded computing, Micro Electro Mechanical Systems (MEMS), broadband and mobile networking, distributed cloud computing, advanced distributed database architectures, cutting-edge web and mobile user interfaces and deep enterprise integrations, all converge. Confluence of low cost microprocessors, ubiquitous WiFi and internet and shrinking devices allowed new categories of on-line technologies. Wearables such as smart watches and fitness bands are excellent examples of IoT In this trend of developments, ECG sensors in the body area electronics, in the form IoT would give advantage in monitoring cardiac patients and provide assisted living with early warnings.

There has been shift in Architectures, from central systems like cloud to edge, in which intelligence is pushed to edge from the central systems, localizing certain kinds of analysis and decision making. Edge computing enables quicker response times, unencumbered by network latency, as well as reduced traffic, 
selectively replaying the appropriate data to the central system. This paradigm shift to Edge computing or FoG computing is championed by some of the biggest IoT technology players including Cisco, IBM and Dell.

ECG Sensor IoT, for realizing the deviation detection objectives, evolved has the following pictorial depiction describing the key elements resident in an edge device (IoT), which is distributed in the form of Body area electronics and cellular mobile stack, connectable over IP net shown in Fig 1.

\begin{tabular}{|c|}
\hline $\begin{array}{c}\text { Long-Haul Communication (IPV6 + Bluetooth/USB } \\
\text { Comm. of IoT from Body Area Electronics) }\end{array}$ \\
\hline ECG Agent \\
\hline Controller \\
\hline ECG Sensor \\
\hline Actuator \\
\hline ECG Internet of Things (IoT) \\
\hline
\end{tabular}

Fig 1 ECG IoT Stack

ECG Sensor:

It is the front end eye and ear of the ECG system with Wavelet Transform analysis [ ], in the subsequent stages of mobile phone or a doctor's central system. A Flexible ECG amplifier sensor patch design with printable Thin Film Transistors (TFTs) has been conceived and published [8].

\section{Actuator:}

Subsequent to Digital processing of the Electro Cardiogram, for it's specific deviation, if any, an early warning is provided by the actuator in the patch, sticked to the body. This patch is in the form of Peizo speaker, printed using Peizo electric material inks.

\section{Controller:}

The next layer ideally is an ECG stack in the controller, a hardware with embedded software component that interacts electrically or logically with sensors and actuators. Here, the actuator could be printable electronic peizo electric speaker or vibrator, in the front end patch, to wake up a sleeping cardiac patent, when gross deviations of ECG signal are detected. In ECG IoT, it is merged in other long haul communication block and exists, logically as a separate block.

\section{ECG Agent:}

The ECG Agent is responsible for transporting digitized ECG data with command codes for 'IoT Data Exchange Protocol Request', 'IoT Deselect Request', 'IoT Release Request' by Cellular Mobile Phone and ' IoT Data Exchange Protocol Response', ' IoT Deselect Response' , ' IoT Release Response' by the ECG IoT device. The ECG Digitized data samples of size $2 \mathrm{Kbit} / \mathrm{sec}$ is transported by the following Transport Frame Format shown in Fig below

\begin{tabular}{|l|l|l|l|l|l|l|l|l|}
\hline $\begin{array}{l}\text { START } \\
\text { BYTE }=\text { '55' }\end{array}$ & $\begin{array}{l}\text { LENGTH } \\
=255\end{array}$ & $\begin{array}{l}\text { CMD } \\
\text { BYTE 1 }\end{array}$ & $\begin{array}{l}\text { CMD } \\
\text { BYTE 2 }\end{array}$ & $\mathbf{X}_{1}$ & $\mathbf{X}_{2}$ & ---- & $\mathbf{X}_{\mathrm{N}}$ & CRC \\
\hline
\end{tabular}

Fig 2: ECG Agent Frame Format for Transportation and Exchange of Data between Mobile unit and Body area unit of ECG IoT

\section{Electro Cardio Gram (ECG) And Phono Cardio Gram}

Cardiac rhythm is controlled by the pacemaker cells known as SinoAtrial (SA) node. The Electro Cardio Gram (ECG) is representation of the electrical activity of the heart, with P,QRS, T waveform as it's one complete cycle. Whenever SA node fires, $\mathrm{P}$ wave occurs and the impulse triggers atrial contraction; isometric segment PQ interval is the propagation delay, when the impulse travels from atria to ventricles, allowing blood flow to complete in one direction; The QRS complex occurs when the electro conduction impulse spreads to ventricles and triggers ventricular contraction; ST segment is the period when the ventricles are de-polarised and ventricular re-polarisation (relaxation) begins and $\mathrm{T}$ wave represents the return to resting state by the ventricles [1]. The general standardized time intervals of ECG used in the algorithm are depicted below in Fig 3 


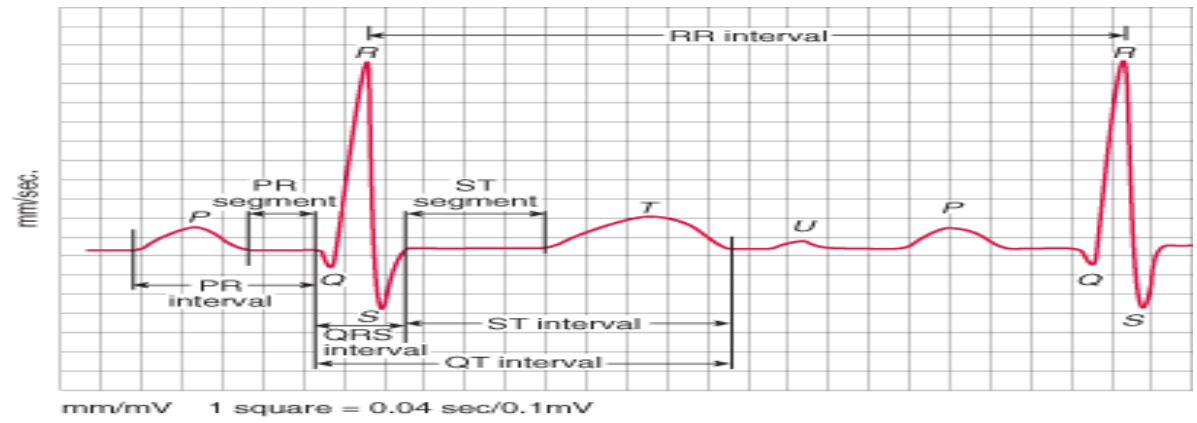

Fig3 Electro Cardio Gram

ECG is normally recorded either using a 12 Channel in hospital or 3 Channel in the field. Hypothetical Einthoven's triangle is commonly used for the electrode set up. The set up requires four limb electrodes placed on the right and left arms and legs; the fourth limb electrode that is not shown in the diagram is placed on the right leg and serves as reference channel. The top of the triangle forms Lead I, while left forms Lead II and right forms Lead III as shown in Fig. 4 with Lead and Polarity table. The voltage differences between the limb electrodes: Left Arm (LA). Right Arm (RA), and Left Leg (LL) leads are to obtain Leads I, II and III [2] [3]

with the three leads in relationship:

$$
\begin{aligned}
& \mathrm{I}=\mathrm{V}_{\mathrm{LA}}-\mathrm{V}_{\mathrm{RA+-}} \\
& \mathrm{II}=\mathrm{V}_{\mathrm{LL}}-\mathrm{V}_{\mathrm{RA}}{ }^{+-} \\
& \mathrm{III}=\mathrm{V}_{\mathrm{LL}}-\mathrm{V}_{\mathrm{LA}}{ }^{-}
\end{aligned}
$$

$$
\mathrm{II}=\mathrm{III}+\mathrm{I}
$$

These are the essential three leads normally sufficient to detect life threatening abnormal ECG rhythms (arrhythmias) and used in this analysis. The same Einthovan's triangle electrode set up does also give an additional three leads and these augmented limb leads (aVF, aVL, aVR) are contributed by [2] [3]

$$
\begin{aligned}
& \mathrm{aVR}=\mathrm{V}_{\mathrm{RA}}-\left(\mathrm{V}_{\mathrm{LA}}+\mathrm{V}_{\mathrm{LL}}\right) / 2 \\
& \mathrm{aVL}=\mathrm{V}_{\mathrm{LA}}-\left(\mathrm{V}_{\mathrm{RA}}+\mathrm{V}_{\mathrm{LL}}\right) / 2 \\
& \mathrm{a} V \mathrm{VF}=\mathrm{V}_{\mathrm{LL}}-\left(\mathrm{V}_{\mathrm{LA}}+\mathrm{V}_{\mathrm{RA}}\right) / 2
\end{aligned}
$$

Compared to six limb leads, these six pre-cordial leads provide a more detailed view of the heart's electrical activity. These six pre-cordial leads are obtained by placing electrodes on the chest as shown below. The obtained voltages are referenced to hypothetical Wilson's Central Terminal, which is the average of voltages $\mathrm{V}_{\mathrm{LA}}, \mathrm{V}_{\mathrm{RA}}$ and $\mathrm{V}_{\mathrm{LL}}[2]$ :

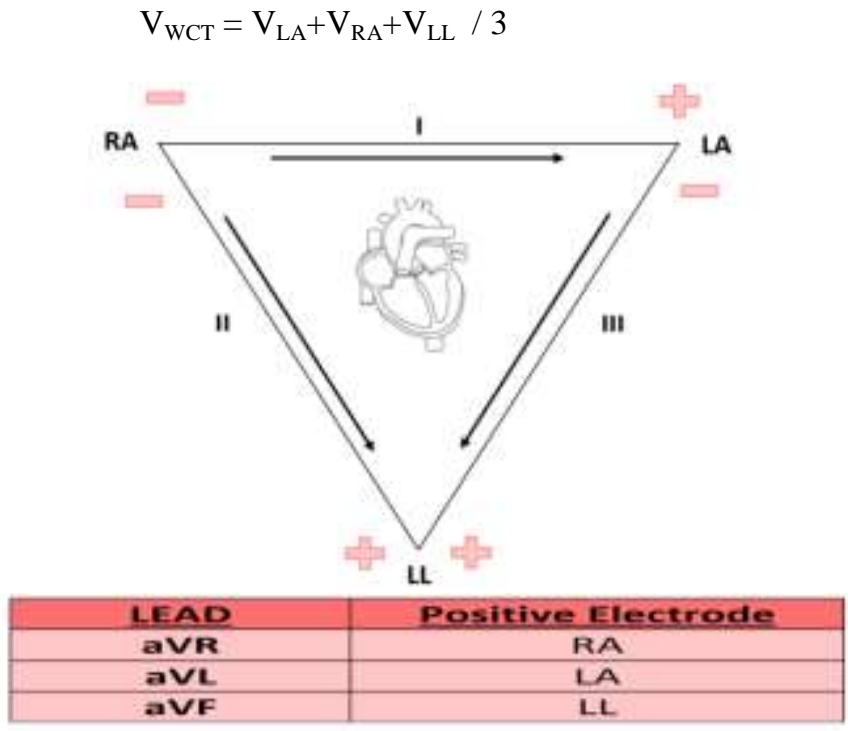

Fig 4

\section{Phonocardiography:}

In addition to electrical activity of the heart, if heamodynamics of the heart muscles are to be indicated, it is Phonocardiography (PCG) that helps. PCG is digital representation of heart sounds. Segmentation and Digital Signal Processing(DSP) of these sonic vibrations involve detection of S1 ( First sound) and S2 (Second sound) 
corresponding to lup and dub and in between heart murmurs, clicks and snaps S3 and S4, which may also indicate mitral valve regurgitation, if it's signatures are detected as work progresses, further.

\section{Body Area Electronics}

In order to continuously log ECG digitized data, a gel free non contact [8] ECG sensor design that couples capacitively with skin [8] is needed. Design exercise undertaken with such purpose earlier [7] is given below in Fig 5. In the present mobile application methodology evolved, for T-wave inversion processing, to detect deviation for various cardiac diagnostics, peizo electric inkjet printed speaker/vibrator, HC05 Blue tooth module and microprocessor board with ADC are also involved [5].

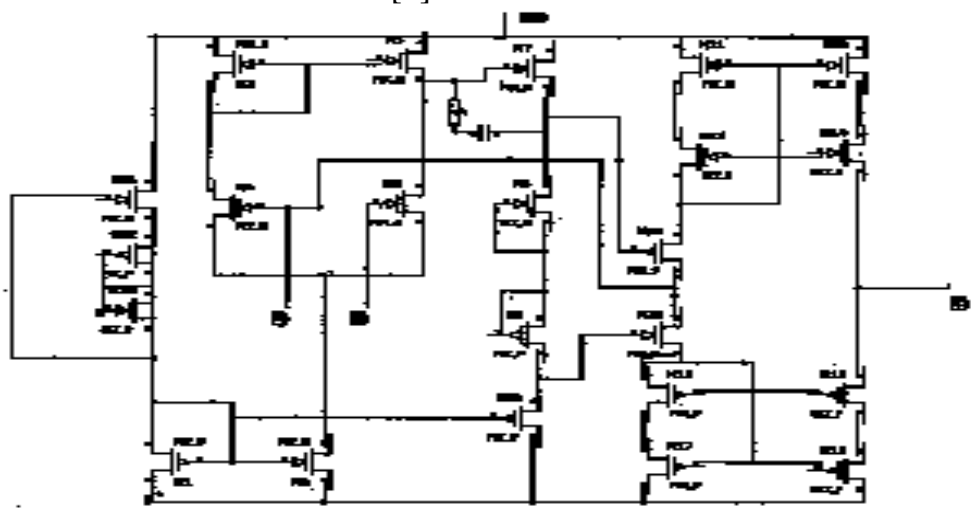

Fig 5 Front end ECG Sensor of IoT Device

Before structural changes in the heart are detected, inverted T-Waves may give an early clue and represent the first and only sign of an inherited heart muscle disease, even in the absence of other deviation features. Deep $\mathrm{T}$-wave inversions of $>=2$ contiguous anterior or lateral leads (but not aVR and III) are of major concerns, as these ECG alterations are a recognised manifestation of hypertrophic cardio myopathy (HCM) and arrhythmogenic right ventricular cardio myopathy (ARVC) [10]. The natural statistics of patients with inverted $\mathrm{T}$ Waves is variable ranging from a normal finding without pathogenic issues to clinical situations causing sudden death related to cardiac or respiratory syndromes [15]. The T wave is normally upright in leads I, II and V2 to V6; inverted in lead aVR; and variable in leads III, aVL, aVF and V1. In general, an inverted T wave in a single lead in one anatomic segment (i.e inferior, lateral or anterior) is unlikely to represent acute pathology, for instance, a single inverted $\mathrm{T}$ wave in either lead III or aVF can be a normal variant.

The causes of $\mathrm{T}$ - wave inversions comprise two categories: Primary T-wave changes and Secondary $\mathrm{T}-$ wave changes [16]. Changes in the morphology of the action potentials, without concurrent changes in the sequence of activation are termed "Primary Changes". Primary T-wave inversions are associated with benign syndromes, such as the persistent juvenile T-wave pattern, including acute coronary ischemic events and cerebral nervous system catastrophe. Secondary Twave changes result from aberrations in ventricular activation, in the context of normal characteristics of action potential; these include bundle-branch blocks, ventricular pre-excitation states, ventricular ectopic beats and left ventricular hypertrophy. In elderly patients, T-wave inversions are associated with coronary artery disease, as seen in myocardial ischemia [17]. Inverted T-waves related to acute coronary syndrome are symmetric in shape [15].

\section{Plotting Electro Cardio Gram (ECG) On Mobile Display}

In the case of a confirmed Cardio Vascular Disease (CVD) patient, detection of changes in Secondary T-wave, setting a threshold is used to generate early warning, even during sleep condition of a heart patient in this methodology. So an algorithm for detecting inversion of 'T-wave' threshold is evolved for mobiles, in continuation to earlier publication [4] in which ECG waveform is segmented in to 410 Cubic Splines for wavelet transform analysis for deviation detection on systems. The algorithm plots data points corresponding to ECG on graph shown on mobile display.

ALGORITHMIC sequence for ' $\mathrm{T}$ wave' deviation detection:

INITIATE Android GraphView ( );

INITIATE Bluetooth ( );

RECEIVE DIGITIZED ECG ( );

$\operatorname{RETURN}\left(\mathrm{X}_{\mathrm{i}}=\mathrm{X}_{1} \mathrm{X}_{2}\right.$

Where $\mathrm{i}=1,2,3$, ------- $\mathrm{N}$ is the length of the dizitized signal with a sample ' $r$ '

DISPLAY_ECG_GRAPH_ON_MOBILE_ONLINE ( ); /using line graph $(\mathrm{Xn}, \mathrm{Xn}+1)$

DETECT_Rpeaks_SETS_OF_ECG $\left(\mathrm{Xp}_{1}, \mathrm{Xp}_{2},-----------, \mathrm{Xpn}\right)>$ Threshold Value

MEASURE_QRS_INTERVAL_AT_EACH_PEAK ( );

RETURN (QRS $\left.{ }_{\text {INT1 }}, \mathrm{QRS}_{\mathrm{INT} 2},---\cdot-\cdot-\cdot-\cdot---, \mathrm{QRS}_{\mathrm{INTN}}\right)$

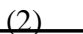




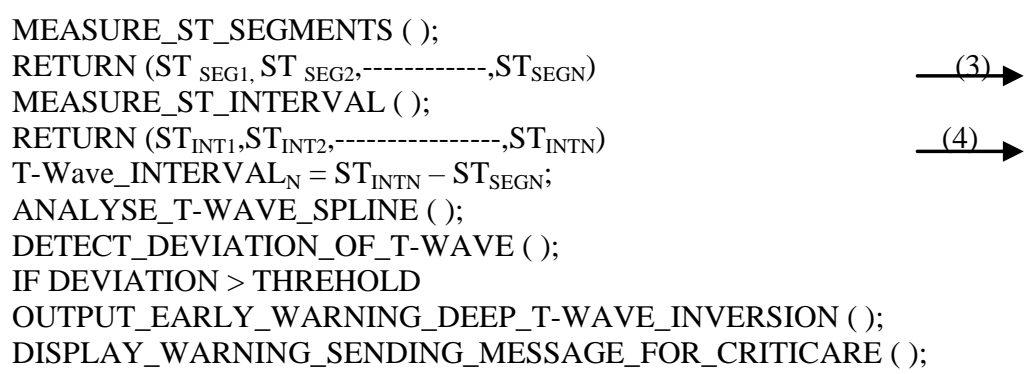

\section{Conclusion}

The algorithm is for detecting non juvenile T-wave inversions, based on secondary wave deep inversions and deviation of T-Wave beyond a threshold, for early warning to the already diagnosed Cardio Vascular Disease (CVD) patient for critical health care, much before structural deterioration of heart anatomy in the case of Myocardial Ishchemia and Cardiomyopathy. The present method evolved, would give early clue and warning to patients leading to hypertrophic cardio myopathy (HCM) and arrhythmogenic right ventricular cardio myopathy (ARVC) for further confirmation. In combination with Phonocardiagram, this type of ECG deviation detection algorithms, segmenting ECG into cubic splines [4] is likely to lead to, many non invasive diagnostics, localisable deviations of ECG, to various heart anatomy structural deviations.

\section{Acknowledgement}

Author would like to thank, Jodhpur National University, India for allowing the work and the support and also National Centre for Flexible Electronics at IIT Kanpur, whose discussions helped, while coordinating with the Centre, as part of his duties.

\section{References}

[1]. B M Beasley, Understanding EKGS: A Practical Approach, $2^{\text {nd }}$ ed.,Pearson Education, 2003

[2]. L Sommo and P Laguna, Bioelectrical Signal Processing in Cardiac and Neurological Applications, Elsevier, 2005

[3]. R M Rangayyan, Biomedical Signal Analysis, A Case Study Approach, IEEE Press, 2002

[4]. M Seshagiri Rao, Dr V S Chouhan, "Wavelet Analysis in Medical Signal Perception for Deviation Detection: A Methodology for ECG Wavelets Deviation Detection", $2^{\text {nd }}$ International Conference on "Emerging Trends \& Technology in Today's Era (ETTTE 2015) ISBN No: 978-81-927182-1-7

[5]. Er M Sesha Giri Rao, "Mobile Application: ECG Signal Processing for Deviation Detection of Heart Rate Variability (HRV)", IOSR Journal of Mobile Computing and Application (IOSR-JMCA), vol X, Issue 5, ver.I (Sep - Oct 2016), e-ISSN:2394-0050,pISSN No.:2394-0042

[6]. Naveen Mysore Balasubramanya, Lutz Lampe, Senior Member IEEE, Gustav Vos and Steve Bennett, “ DRX with Quick Sleeping: A Novel Mechanism for Energy-Efficient IoT Using LTE/LTE-A, IEEE Internet of Things Journal, Vol.3, Np.3, June 2016

[7]. Er M Seshagiri Rao and Dr V S Chouhan, "Epidermal Flexible Printable Non Contact Autonomous ECG Sensor Medical Device Patch for Deviation Detection", IOSR Journal of VLSI and Signal Processing (IOSR-JVSP), vol.6, Issue 3, ver (May - June 2016), e-ISSN:2319-4200, p-ISSN No:2319-4197, DOI: 10.9790/4200-6603

[8]. Thomas J. Sullivan, Stephen R and Gert Cauwenberghs University of California, "A Low-Noise, Non-Contact EEG/ECG Sensor" 1-4244-1525-X/07 IEEE 2007

[9]. Ye Wang, Wei Sun, Hilmi Volkan Demir, "Influence of Channel Layer Thickness on the Electrical Performances of Inkjet-Printed In-Ga-Zn Oxide Thin-Film Transistors" IEEE Trans Electron Devices, vol. 58 no.2, February 2011

[10]. M G Wilson, S Sharma, F Carie et al, "Significance of Deep T-wave Inversions in asymptomatic athletes with normal Cardio Vascular examinations: Practical solutions for managing the diagnostic conundrum", British Journal of Sports Medicine, 2012, DOI. 10.1136/BJSPORTS-2012-090836

[11]. M.SeshaGiri Rao, Dr. V S Chouhan, "Wavelet based Signal Processing for Compression a Methodology for on-line Tele Cardiology", IOSR Journal of VLSI and Signal Processing (IOSR-JVSP) Volume 5, Issue 6, Ver. I (Nov -Dec. 2015), PP 46-51 eISSN: $2319-4200$, p-ISSN No. : $2319-4197$

[12]. Prerana N Gowale, AN Cheeran, Nidhi G Sharma, VJTI, Maharashtra, International Journal of Advanced Research in Computer and Communication Engineering Vol.3, Issues 5, May 2014, ISSN-(Online) 2278 - 1021, ISSN (Print): 2319 - 5940

[13]. Isha V Upganlawar, Harshal Chowan, Dept of Computer Science and Engineering, Wainaganga College of Engineering \& Technology, "Preprocessing of ECG Signals Using Filters", International Journal of Computer Trends and Technology (IJCTT) Volume $11,4^{\text {th }}$ May 2014, ISSN: 2231-2803

[14]. Olivier Meste, Darius Janusek, S Karczmarewicz, A Przybylski, Michal Kania, et al.. Improved robust T-wave alternans detectors. Medical and Biological Engineering and Computing, Springer Verlag, 2015, 53 (4), pp.361-370.

[15]. Simon K Hackett D, Szelier A, SZeprology's A, et al. (1994). "The natural history of postischemic T- wave inversion: a predictor of poor short term prognosis of Coronary Artery Dis 5:937-942

[16]. Salah AM Said, Rene Bloo, Ramon de Nooijer, et al, "Cardiac and non-Cardiac causes of T - wave inversion in the Pre-cardial leads in adult subjects; A Dutch case series and review of the literature", World Journal of Cardiology 2015, February 26; 7(2); 86100, ISSN 1949-8462, DOI:10.4330/wjc.v7.i2.86

[17]. Massimo Bolognesi, "The grey zone of physicians: Negative T waves on ECGs pre-cordial leads", Department of Internal General Medicine, General Practitioner - AST, 112 Cesna Via Ungaretti - 49447521 Cesena, Italy ISSN:2059-0393, Clin Case Rep Rev, 2015 DOI: 10-15761/ccRR.1000145, volume 1(7):136-138 